\title{
The Response of Arterioles in Skeletal Muscle Grafts to Vasoactive Agents
}

\author{
Harold W. Burton and John A. Faulkner \\ Department of Physiology, The University of Michigan Medical School, \\ Ann Arbor, Michigan 48109
}

Received March 6, 1986

\begin{abstract}
Arteriolar responses to the vasodilator adenosine and to the vasoconstrictor norepinephrine (NE) were examined in small bundles of extensor digitorum longus muscle grafted onto the cheek pouches of hamsters. Responses of arterioles to topically applied adenosine or NE were measured from 30 to 180 days after grafting and compared with the response of arterioles in control cheek pouches. Verapamil and potassium chloride $(\mathrm{KCl})$ were applied to 120- and 180-day grafts to determine the response of arterioles to vasoactive agents not mediated by receptors. Arterioles in grafted muscle did not respond to adenosine until 60 days. The response increased with time but was significantly less than the control value even after 180 days. Arterioles in grafts did not respond to NE until 90 days. At both 90 and 120 days the degree of constriction in response to NE was not uniform along the length of a given arteriole (punctate response). By 180 days, the response of a given arteriole to NE was uniform but significantly less than the control value. In 120- and 180day grafts the responses of arterioles to verapamil and $\mathrm{KCl}$ were similar to the responses of arterioles to adenosine and NE, respectively. We conclude that the diminished response of arterioles in small EDL muscle grafts to vasoactive agents may be caused by either a structural or a functional impairment in the smooth muscle layer of arterioles. 1987 Academic Press, Inc.
\end{abstract}

\section{INTRODUCTION}

When skeletal muscles are autografted by standard operative procedures, all neural and vascular connections are severed and the transplanted muscle revascularizes and reinnervates spontaneously (Carlson et al., 1979). Because the graft is ischemic for a period of days or weeks (Hansen-Smith et al., 1980; White et al., 1981), the muscle fibers degenerate and subsequently regenerate from a population of satellite cells (Snow, 1977). The revascularization of grafted skeletal muscle has been described previously (Hansen-Smith et al., 1980; Faulkner et al., 1983), but the reactivity of blood vessels in grafts to vasoactive agents has not been examined.

In extensor digitorum longus (EDL) muscle grafts in cats, White et al. (1981) reported an increase in resting blood flow, and then a decrease to control levels. Apparently, the regenerated blood vessels had a decreased vasomotor tone initially but this deficiency disappeared as the graft reached maturity. In response to 
twitch contractions, maximum blood flow in the mature grafts was reached at a lower twitch frequency and was of a greater magnitude when normalized per $100 \mathrm{~g}$ of contractile protein. Thus, arterioles in mature standard muscle grafts appear to vasodilate maximally in response to low-frequency twitch contractions. The abnormal function may be due to either a decreased smooth muscle tone or an increased sensitivity of arterioles to locally released vasodilators. Irregularities observed in the smooth muscle layers of regenerated arterioles of grafted muscle (Hansen-Smith et al., 1980) may also contribute to the inability of arterioles in grafts to control blood flow.

The purpose of this study was to examine, in grafted skeletal muscles, the effect of two vasoactive agents, adenosine and norepinephrine (NE), that act on arteriolar diameter through specific receptors. Two hypotheses are tested: compared with control arterioles in the hamster cheek pouch, arterioles in grafted EDL muscles have (1) a greater sensitivity to topically applied adenosine and (2) a decreased sensitivity to NE. In addition, the hypothesis that impairments in vascular reactivity are due to modifications in the function of the smooth muscle layer of arterioles is tested by comparing the maximum response of arterioles in grafted muscle to adenosine and NE with the response to the nonspecific vasoactive agents verapamil and potassium.

\section{MATERIALS AND METHODS}

A model in which small bundles of skeletal muscle were grafted onto the hamster cheek pouch was selected for these experiments. This model provided adequate video resolution for the measurement of changes in the diameter of arterioles in grafts following the topical application of vasoactive agents. Syrian Golden Hamsters weighing $100-110 \mathrm{~g}$ were anesthetized with a sterile intraperitoneal injection of sodium pentobarbital $(0.1 \mathrm{mg} / \mathrm{g}$ body wt). A total of 20 control and 52 experimental animals were used in this study.

The procedure for grafting EDL muscle bundles onto the hamster cheek pouch was a modified version of that described by Faulkner et al. (1983). The EDL muscle was removed from the left hindlimb under sterile conditions and divided into six to eight bundles of approximately 20 to 30 intact fibers. Two muscle bundles were grafted onto the left cheek pouch of the donor animal and onto the cheek pouches of two to three additional animals. To prepare the cheek pouch for grafting, a plastic disc, $3 \mathrm{~cm}$ in diameter, was attached to a springloaded holder (Greenblatt et al., 1969) and inserted into the cheek pouch. An incision, approximately $4 \mathrm{~cm}$ in length, was made through the skin over the plastic disc in an anteroposterior direction. The incision was spread apart and the skin was retracted over the disc so that the surface of the cheek pouch was exposed. The mucoareolar membrane was removed. Each bundle of EDL muscle fibers was soaked in $0.75 \%$ bupivicaine for $10 \mathrm{~min}$ to ensure that all fibers were destroyed and that the muscle would regenerate from surviving satellite cells (Carlson, 1976). Bupivicaine is a myotoxic agent that causes degeneration of the muscle fiber but leaves the satellite cells and the original basal lamina of the fiber intact. Grafts were sutured onto the cheek pouch on top of, and perpendicular to, the skeletal muscle fibers located in the proximal region of the cheek pouch (Duling, 1973). This orientation permitted differentiation between fibers in the 
graft and in the cheek pouch. The holder and plastic disc were removed and the incision over the cheek pouch was closed with 5-0 suture. Betadine solution was applied to the area of the incision.

Responses of arterioles in the grafts to topical application of either $10^{-4} \mathrm{M}$ adenosine or $10^{-6} M$ NE were measured in different groups of animals at either $30,60,90,120$, or 180 days postgrafting. Grafts in each of eight animals were studied at each time period. These results were compared with the average response of arterioles in the proximal region of the cheek pouch in a group of control hamsters $(n=15)$. In a separate experiment, the response of arterioles in grafts to $10^{-6} M$ verapamil and $10^{-1} M \mathrm{KCl}$ was measured at 120 and 180 days $(n=6)$ and compared with the response of arterioles in control cheek pouches $(n=5)$. To determine whether vascular reactivity of control arterioles varied during the 180-day experimental period, the response of two arterioles to $10^{-4} M$ adenosine and to $10^{-6} M \mathrm{NE}$ was measured in the cheek pouches of each of four experimental hamsters 180 days after grafting. No significant difference was observed between the responses of control arterioles at these two time periods.

Concentrations of $10^{-4} M$ adenosine, $10^{-5} M \mathrm{NE}, 10^{-6} M$ verapamil, and $10^{-1}$ $M \mathrm{KCl}$ produced the maximum responses in control arterioles. The arterioles returned to their original diameter within 15 to $20 \mathrm{~min}$ after the application of either adenosine or $\mathrm{KCl}$ and within times ranging from 25 to 45 min after the application of $10^{-6} M$ verapamil or $10^{-5} M$ NE. Arterioles tended to lose their vascular tone after only a few applications of $10^{-5} M$ NE. For this reason a concentration of $10^{-6} M \mathrm{NE}$ was used to examine arteriolar response in all grafts and a concentration of $10^{-5} M \mathrm{NE}$ was used only occasionally at each time period. A concentration of $10^{-6} M \mathrm{NE}$ elicited an average decrease in control arteriolar diameter of approximately $60 \%$ and the vessel diameter returned to initial levels within 15 to 20 min.

To examine the responses of arterioles to the vasoactive agents, a hamster was anesthetized and cannulae were inserted into the trachea and the right femoral vein. The cheek pouch was prepared for viewing by intravital microscopy as described previously except that the plastic disc was left inside the cheek pouch. The hamster was transferred to the stage of an intravital microscope (Wayland and Frasher, 1971). Before each experiment, the muscle grafts were checked for viability either through direct electrical stimulation or through stimulation of the skeletal muscle layer underneath the graft. By touching the muscle layer with a pair of metal forceps, a twitch could be elicited in the graft. Only those grafts that responded to stimulation by twitching were used in this study. In 11 of the 52 experimental animals, neither of the two grafts responded to stimulation. No data were collected on these grafts.

To maintain body temperature at approximately $37^{\circ}$ the hamster was placed on a heated pad. The plastic disc was secured to a plexiglass holder, forming a well $0.5 \mathrm{~cm}$ deep and $2.5 \mathrm{~cm}$ in diameter, with the cheek pouch at the bottom of the well. A $0.5-\mathrm{cm}$-wide groove in the side of the well allowed the suffusate to run off the cheek pouch. Suffusate at a temperature of $37^{\circ}$ and a $\mathrm{pH}$ of 7.4 was delivered from a $50-\mathrm{ml}$ reservoir through an 18-gauge blunted needle to bathe the surface of the cheek pouch. The suffusate was a bicarbonate-buffered salt solution gassed with $5 \% \mathrm{CO}_{2}$ in nitrogen. The suffusate had a millimolar composition 
of $\mathrm{NaCl} 130.9, \mathrm{KCl} 4.7, \mathrm{CaCl}_{2} 2.0, \mathrm{MgSO}_{4} 1.2$, and $\mathrm{NaHCO}_{3} 20.0$. The flow rate of the suffusate of 5-6 $\mathrm{ml} / \mathrm{min}$ was adjusted to provide a cheek pouch temperature of approximately $35^{\circ}$, measured by a bead thermistor placed directly on the cheek pouch. The suffusate was withdrawn by aspiration through the groove in the side of the well. Anesthesia was maintained at a constant level by infusion of a dilute solution of pentobarbital sodium $(9 \mathrm{mg} / \mathrm{ml})$ into the femoral vein at a rate of $0.5 \mathrm{ml} / \mathrm{hr}$. The hamster breathed a free-flowing gas mixture of $30 \%$ oxygen in nitrogen spontaneously through a tracheal cannula. The high oxygen concentration of the inspired gas ensured that arterial $P_{\mathrm{O}_{2}}$ remained at a level that would not affect vascular tone. Arterial $P_{\mathrm{O}_{2}}$ of hamsters breathing air is 50$60 \mathrm{~mm} \mathrm{Hg}$, which may affect vascular tone of blood vessels (Robert Hester, University of Mississippi, personal communication).

During four preliminary experiments blood pressure and blood gases were recorded from the right carotid artery. Blood pressure was recorded every 30 min and a blood sample of $0.5 \mathrm{cc}$ was withdrawn every $60 \mathrm{~min}$ for $4 \mathrm{hr}$. The mean blood pressure of $108 \pm 3 \mathrm{~mm} \mathrm{Hg}$ did not vary significantly from the beginning to the end of the experiment. Blood $P_{\mathrm{O}_{2}}$ was $87 \pm 4 \mathrm{~mm} \mathrm{Hg}$ at the beginning and $80 \pm 3 \mathrm{~mm} \mathrm{Hg}$ at the end of the 4-hr period. This range of arterial $P_{\mathrm{O}_{2}}$ is slightly higher than the values of $74 \pm 5 \mathrm{~mm} \mathrm{Hg}$ measured in unanesthetized hamsters (O'Brien et al., 1979; Lucey et al., 1980). The difference is unlikely to affect resting arteriolar diameter (Duling, 1972).

Experimental protocol. In each experiment, the vasoactive agent was added to the $50-\mathrm{ml}$ reservoir to give the designated final concentration. Changes in arteriolar diameter were measured directly from a video monitor and the maximum change in diameter was recorded. The initial and final diameters of arterioles were calculated based on prior calibration using a stage micrometer. After each administration of either adenosine or NE, the reservoir was flushed three times with suffusate. Enough time, at least $15 \mathrm{~min}$, was allowed between administrations to allow the arteriole to return to its original diameter. Since smaller arterioles generally show a greater sensitivity to NE than do larger arterioles (Duling et al., 1968), variability among arterioles was minimized by using arterioles between 10 and $30 \mu \mathrm{m}$ in diameter.

Since vascular tone was not present in some preparations, each experiment began with an administration of $10^{-4} M$ adenosine to confirm that the artcriolc was reactive. In experiments in which arterioles in muscle grafts were being examined, the test for reactivity was conducted using arterioles in the cheek pouch. If arterioles in the cheek pouch did not respond to $10^{-4} M$ adenosine with greater than an $80 \%$ increase in arteriolar diameter, the preparation was rejected. Data from 7 of the control animals and 10 of the experimental animals were rejected on the basis of a subnormal response to adenosine.

All data are presented as means \pm SEM. Significant differences between the mean responses of arterioles in grafts and the mean responses of artcrioles in control animals were determined by a Student $t$ test for small samples (Snedecor and Cochran, 1967). 


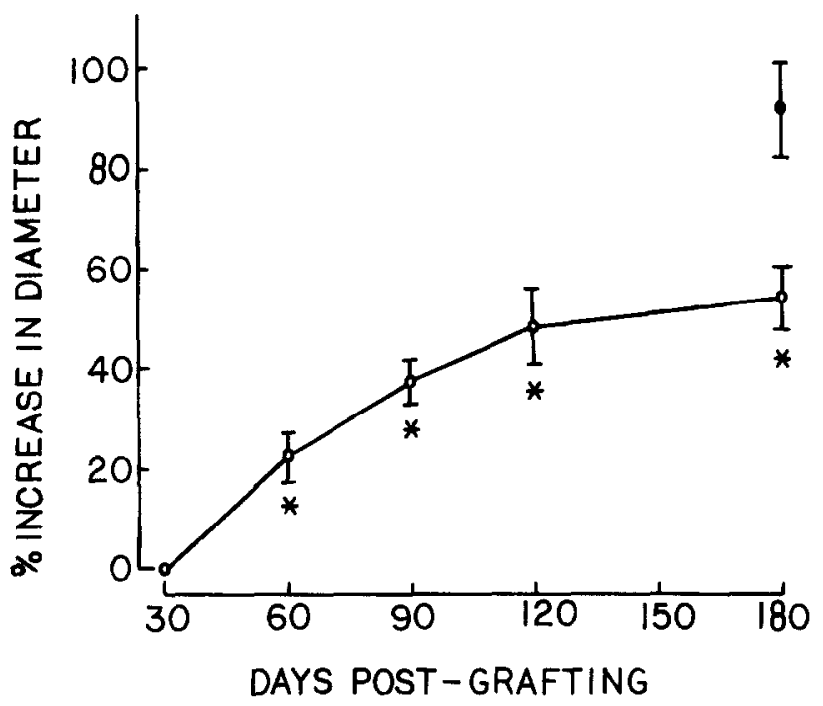

FIG. 1. Responses of arterioles in control cheek pouches $(\bullet)(n=12)$ and of arterioles in grafted muscle between 30 and 180 days $(O)$ when $10^{-4} M$ adenosine was suffused onto the hamster cheek pouch. Values given are means \pm SEM. For arterioles in grafts, $n=4$ at 30, 60, and 90 days, $n=6$ at 120 days, and $n=12$ at 180 days. $*$ Significantly different from control $(P<0.01)$.

\section{RESULTS}

The response of control arterioles in cheek pouches and of arterioles in grafted EDL muscles to $10^{-4} M$ adenosine is presented in Fig 1. Arterioles in control cheek pouches had an average initial diameter of $23 \pm 4 \mu \mathrm{m}$ and increased in diameter by $93 \pm 8 \%$ when adenosine was applied. Arterioles in grafted muscle did not respond to adenosine until 60 days postgrafting and the response was significantly less than the control value $(P<0.01)$. A gradual increase in the response to adenosine was observed as the number of days postgrafting increased, but the average increase in diameter at 180 days was only $54 \%$. This response was significantly less than the control value $(P<0.01)$.

The responses of arterioles in control cheek pouches and arterioles in grafted muscle to $10^{-6}$ and $10^{-5} M$ NE are presented in Table 1 . When arterioles in control cheek pouches reached their maximum response to $10^{-6} M \mathrm{NE}$, blood flow was either intermittent or stopped completely. Blood flow stopped completely in response to $10^{-5} M$ NE. Arterioles in 30- and 60-day grafts did not constrict when either $10^{-6}$ or $10^{-5} M$ NE was applied, but blood flow through the graft was affected by the constriction of arterioles in the cheek pouch. Under these circumstances, the control of blood flow resided in the cheek pouch arterioles outside of the graft. In 90- and 120-day grafts, NE caused a segmented or punctate response in most arterioles. For example, the diameter of one segment of an arteriole in a 120-day graft decreased by $40 \%$ of resting diameter while an adjacent segment decreased by $70 \%$. This segmented appearance of the arteriole, produced by variable responses of different sections to NE, was present in 8 of 10 arterioles in 90- and 120-day grafts that were exposed to $10^{-6} \mathrm{MNE}$ and in 6 of 7 arterioles in 90- and 120-day grafts that were exposed to $10^{-5} M$ NE. The response was uniform along the length of the other three arterioles. Arterioles in 90- and 120- 
TABLE 1

Response of Arterioles in Control Cheek Pouches and of Arterioles in Grafted Muscle to $10^{-5} M$ AND To $10^{-6} M$ NE (MEANS \pm SEM)

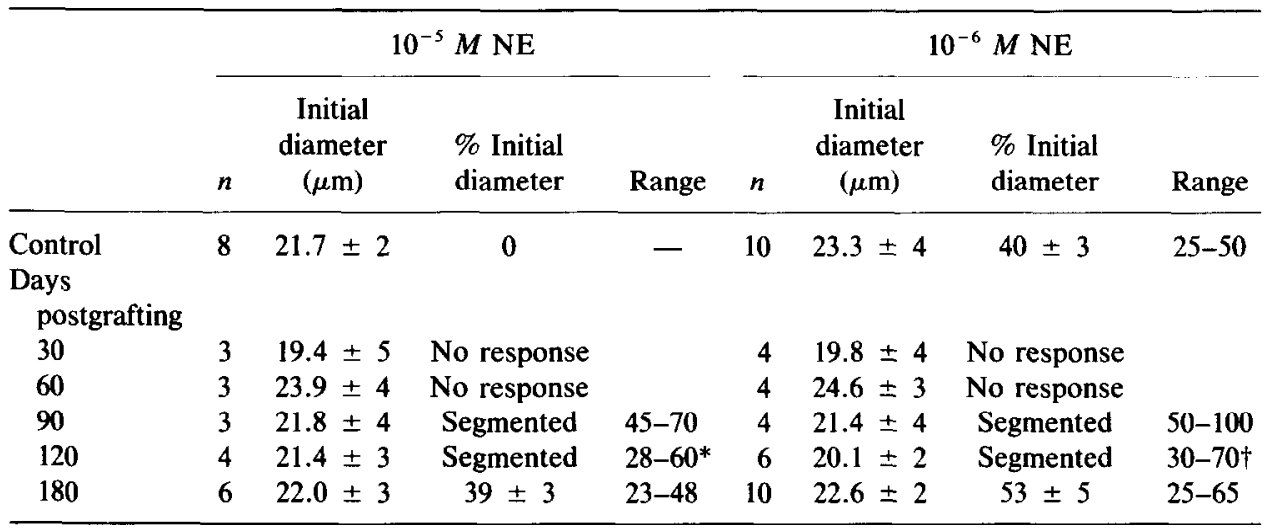

* One arteriole constricted uniformly along its length to $32 \%$ of the initial diameter.

$\dagger$ Two arterioles constricted uniformly to 61 and $53 \%$ of the initial diameters, respectively.

day grafts showed a wide range of responses, but the constriction of arterioles in the 120-day grafts was generally greater than that in 90-day grafts. Although the segmented arteriolar response to NE was absent in 180-day grafts, the average decrease in diameter was only $79 \%$ of the control response to $10^{-6} M \mathrm{NE}(P<$ $0.05)$ and $61 \%$ of the control response to $10^{-5} M$ NE $(P<0.01)$.

The responses of control arterioles and of those in 180-day grafts to different concentrations of adenosine and NE are presented in Figs. 2 and 3. At a con-

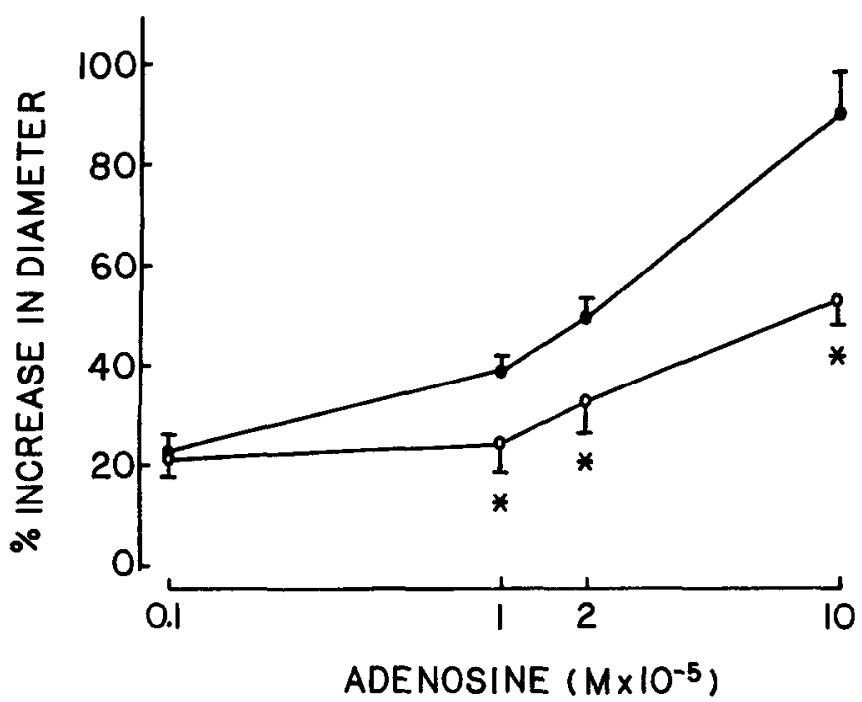

FIG. 2. Responses of arterioles to different concentrations of adenosine observed in control cheek pouches ( $\bullet$ ) and in 180-day grafts ( $(0)$. Each dose was applied randomly. At each concentration of adenosine, $n=8$ for both arterioles in control cheek pouches and arterioles in grafts, except for $10^{-4} M$ adenosine where $n=12$. Values given are means \pm SEM. ${ }^{*}$ Significantly different from control $(P<0.01)$. 


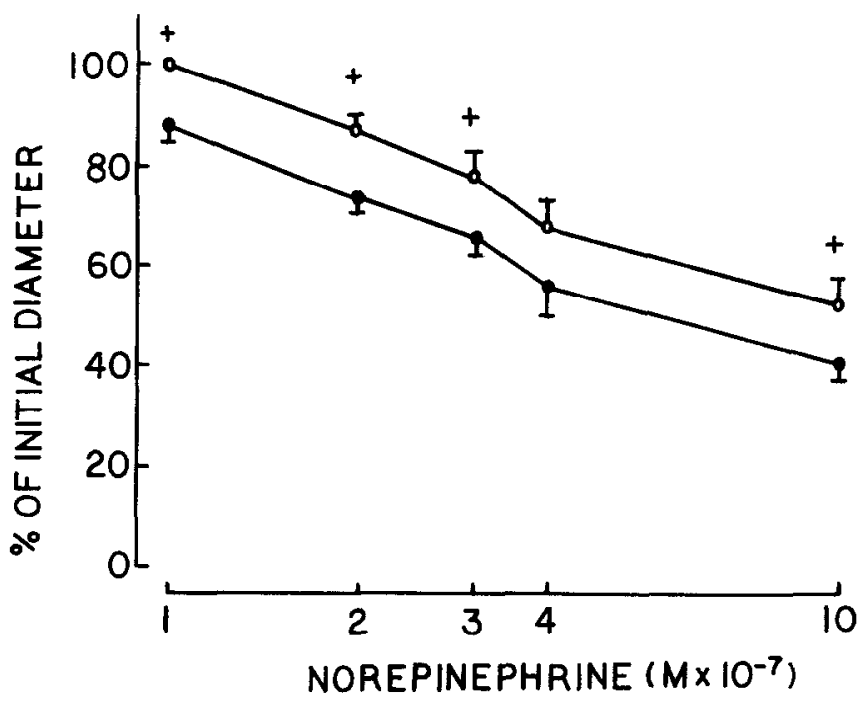

Fig. 3. Responses of arterioles to different concentrations of NF. observed in arterioles in control cheek pouches $(\bullet)$ and in 180-day grafts $(O)$. Each dose was applied randomly. At each concentration of NE, $n=8$ for both control arterioles and arterioles in grafts, except for the control $10^{-6} M \mathrm{NE}$ where $n=10$. Values given are means \pm SEM. ${ }^{+}$Significantly different from control $(P<0.05)$.

centration of $10^{-6} M$ adenosine, the response of arterioles in grafts was not different from the control value. The responses of arterioles in grafts was significantly less than control responses at all other concentrations $(P<0.01)$. At each concentration of NE except $4 \times 10^{-7} \mathrm{M}$, the change in diameter of arterioles in grafts was significantly less than the control response $(P<0.05)$.

To determine whether the diminished response of arterioles in grafts to adenosine and NE was the result of impaired receptor function, the nonspecific vasodilator verapamil and the nonspecific vasoconstrictor $\mathrm{K}^{+}$(added to the suffusate as $\mathrm{KCl}$ ) were topically applied to 120 - and 180-day grafts. These results are presented in Table 2. In two arterioles in each of four cheek pouches, the average increase

TABLE 2

Comparison of the Maximum Responses of Arterioles in Control Cheex Pouches and in 120and 180-Day Grafts to Verapamil, adenosine, Potassium Chloride, and Norepinephrine (Means \pm SEM)

\begin{tabular}{|c|c|c|c|c|c|c|c|c|c|}
\hline & \multicolumn{3}{|c|}{ Control } & \multicolumn{3}{|c|}{ Graft (120 days) } & \multicolumn{3}{|c|}{ Graft (180 days) } \\
\hline & $n$ & $\begin{array}{c}\text { Initial } \\
\text { diameter }\end{array}$ & $\begin{array}{l}\% \text { Initial } \\
\text { diameter }\end{array}$ & $n$ & $\begin{array}{c}\text { Initial } \\
\text { diameter }\end{array}$ & $\begin{array}{l}\% \text { Initial } \\
\text { diameter }\end{array}$ & $n$ & $\begin{array}{c}\text { Initial } \\
\text { diameter }\end{array}$ & $\begin{array}{l}\% \text { Initial } \\
\text { diameter }\end{array}$ \\
\hline $\begin{array}{l}\text { Verapamil } \\
\left(10^{-6} M\right)\end{array}$ & 8 & $20.6 \pm 2$ & $197 \pm 8$ & 6 & $22.0 \pm 2$ & $140 \pm 7$ & 6 & $21.8 \pm 3$ & $162 \pm 6$ \\
\hline Adenosine & & & & & & & 0 & $21.0+3$ & $102-0$ \\
\hline$\left(10^{-4} M\right)$ & 12 & $23.0 \pm 4$ & $193 \pm 8$ & 6 & $20.2 \pm 3$ & $145 \pm 6$ & 12 & $23.6 \pm 4$ & $154 \pm 5$ \\
\hline $\mathrm{KCl}\left(10^{-1} M\right)$ & 8 & $23.6 \pm 3$ & 0 & 4 & $22.2 \pm 2$ & Segmented & 6 & $21.8 \pm 3$ & $45 \pm 7$ \\
\hline NE $\left(10^{-5} M\right)$ & 8 & $21.7 \pm 2$ & 0 & 4 & $21.4 \pm 3$ & Segmented & 6 & $22.0 \pm 3$ & $39 \pm 3$ \\
\hline
\end{tabular}


in diameter in response to $10^{-6} M$ verapamil was $97 \pm 8 \%$. The increase in diameter in response to verapamil was not significantly different from the increase in diameter of arterioles in control cheek pouches in response to $10^{-4} M$ adenosine. Similarly, the responses of arterioles in 120- or 180-day grafts to $10^{-6} M$ verapamil and $10^{-4} M$ adenosine were not significantly different. There was no further increase in the response of arterioles in 180-day grafts when the concentration of either adenosine or verapamil was increased, indicating that maximum responses had been reached at concentrations of $10^{-4} M$ adenosine and $10^{-6} M$ verapamil.

Seven of eight arterioles in control cheek pouches were completely occluded by addition of $10^{-1} M \mathrm{KCl}$ to the suffusate. In the other arteriole the diameter was decreased to $12 \%$ of the initial diameter. There was no difference between the responses of arterioles to $10^{-5} M \mathrm{NE}$ and $10^{-1} M \mathrm{KCl}$ in either 120- or 180day grafts. There was no further increase in the response of arterioles in 180day grafts when the concentration of either $\mathrm{NE}$ or $\mathrm{KCl}$ was increased.

\section{DISCUSSION}

Skeletal muscles that are transplanted to a different site without vascular or neural anastomoses adapt to the innervation and functional requirements of that site (Gutmann and Carlson, 1975). In our experiments, small bundles of EDL muscle fibers were transplanted onto the cheek pouch. Under these circumstances, the skeletal muscle fibers that regenerate are skeletal muscle fibers with the characteristics of fibers in the cheek pouch. Some of the endothelial cells survive this grafting procedure but smooth muscle cells do not (Faulkner et al., 1983). Revascularization of the small grafts in the cheek pouch appear to result from growth of endothelial cells out from the graft with subsequent anastomosis with a vessel in the cheek pouch (Faulkner et al. 1983; Weiss and Faulkner, 1983). Conversely, the smooth muscle cell layer of blood vessels in the graft must develop from the migration and division of smooth muscle cells that were originally from the cheek pouch. Based on this series of events, arterioles in the cheek pouch were used as controls rather than arterioles in EDL muscle.

Compared with arterioles in the proximal region of the cheek pouch, arterioles in bundles of muscle grafts have a diminished response to the vasodilators adenosine and verapamil and to the vasoconstrictors $\mathrm{NE}$ and $\mathrm{K}^{+}$. This indicates an impaired ability of the vascular bed of standard free muscle grafts to regulate blood flow. Verapamil and $\mathrm{K}^{+}$are nonspecific vasoactive agents that change the lumen diameter of arterioles by acting directly on the smooth muscle, whereas adenosine and NE interact with specific receptors with subsequent activation of second messenger systems. The similarity of the responses of arterioles in 120- and 180day grafts to verapamil and adenosine and to $\mathrm{K}^{+}$and $\mathrm{NE}$, respectively, indicates that the diminished response of arterioles in grafts may be due to abnormalities in the vascular smooth muscle layer rather than a dysfunction of the receptormediated responses. A broad spectrum of structural abnormalities has been observed in the smooth muscle coat of blood vessels in EDL muscle grafts in rats (Hansen-Smith et al., 1980).

Direct evidence is lacking, but the punctate or segmented arteriolar response to $\mathrm{NE}$ at 90 and 120 days may have resulted either from a discontinuity in the smooth muscle layer or from irregularities in the organization of the smooth 
muscle cell layer (Hansen-Smith et al., 1980). At 180 days postgrafting, arterioles constricted uniformly along the length of the arteriole when NE was applied, indicating that the abnormal response to NE had disappeared.

White et al. (1981) reported that the resting blood flow of standard EDL muscle grafts in cats increased fourfold over control values during the first months after grafting but eventually returned to control levels. The grafts used in the present study were much smaller, but the results indicate that the increase in resting blood flow may have been due to a loss of vasomotor tone caused by either a complete absence of a response, or a diminished response, to NE. Although the time course of events is different, our obscrvation that the response of arterioles in the cheek pouch grafts to NE returned gradually and was near control values by 180 days postgrafting is consistent with the gradual return of resting blood flow to control values observed in the larger EDL grafts in cats. Whether the response of small grafts in the hamster cheek pouch to NE returns to control levels at any time past 180 days cannot be determined at this time.

White et al. (1981) also observed that blood flow in EDL grafts in cats was poorly regulated in response to twitch contractions. A maximum blood flow was reached at a frequency of only $1 \mathrm{~Hz}$, whereas in control EDL muscles blood flow reached a maximum value at $4 \mathrm{~Hz}$. The hypothesis that the high flow rate in grafts in response to a low metabolic challenge was due to an increased sensitivity to locally released vasodilators is not supported by the present data. Rather, the present study indicates a diminished response of arterioles to the vasodilators adenosine and verapamil. Decreased vascular tone resulting from a decreased sensitivity to NE may be responsible for the diminished response of arterioles to vasodilators and may contribute to the high blood flow rate observed in grafts in response to low-frequency twitch contractions.

Since arterioles in grafted EDL muscle in this study showed a decreased response to adenosine, even after 180 days postgrafting, we reject the hypothesis that arterioles in grafts are more sensitive to adenosine than are those in controls. The hypothesis that arterioles in grafts are less sensitive to NE was accepted. Although the exact mechanism remains uncertain, the decreased response of arterioles in grafted muscle to NE appears related to a dysfunction in the smooth muscle layer. The decreased response of arterioles to adenosine may be caused by a decreased vasomotor tone and a reduction in the capacity to vasodilate. The response of arterioles to a variety of vasoactive substances needs to be examined before arteriolar function in grafted skeletal muscle can be fully elucidated.

\section{ACKNOWLEDGMENTS}

The authors thank Brian Duling for his valuable input. This study was supported by NIH Grants DE 07687 and HL 34164. Harold Burton is a research fellow of the Canadian Heart Foundation.

\section{REFERENCES}

Carlson, B. M. (1976). A quantitative study of muscle fiber survival and regeneration in normal, predenervated, and Marcaine-treated free muscle grafts in the rat. Exp. Neurol. 52, 421-432.

Carlson, B. M. Hansen-Smith, F. M., and Magon, D. K. (1979). The life history of a free muscle graft. In "Musclc Regeneration', (A. Mauro, Ed.), pp. 493-507. Raven Press, New York.

Duling, B. R. (1972). Microvascular responses to alterations in oxygen tension. Circ. Res. $31,1972$. 
Duling, B. R. (1973). The preparation and use of the hamster cheek pouch for studies of the microcirculation. Microvasc. Res. 5, 423-429.

DuLING, B. R., BERNE, R. M., AND Born, G. V. R. (1968). Microiontophoretic application of vasoactive agents to the microcirculation of the hamster cheek pouch. Microvasc. Res. 1, 158-173.

Faulkner, J. A., Weiss, S. W., and McGeachie, J. K. (1983). Revascularization of skeletal muscle transplanted into the Hamster cheek pouch: Intravital and light microscopy. Microvasc. Res. 26, 49-64.

Greenblatt, M., Choudari, K. V. R., Sanders, A. G., and Shubik, P. (1969). Mammalian microcirculation in the living animal: Methodologic considerations. Microvasc. Res. 1, 420-432.

Gutmann, E., And Carlson, B. M. (1975). Contractile and histochemical properties of regenerating cross-transplanted fast and slow muscles in the rat. Pflugers Arch. 353, 227-239.

HANSEN-SMith, F. M., Carlson, B. M., AND IRwin, K. L. (1980). Revascularization of the freely grafted extensor digitorum longus muscle in the rat. Amer. J. Anat. 158, 65-82.

Lucey, E. C., O'Brien, J. J., Periere, W., Jr., And Snider, G. L. (1980). Arterial blood gas values in emphysematous hamsters. Amer. Rev. Respir. Dis. 121, 83-89.

O'Brien, J. J., LuceY, E. C. AND Snider, G. L. (1979). Arterial blood gases in normal hamsters at rest and during exercise. J. Appl. Physiol. 46, 806-810.

Snedecor, G. W., and Cochran, W. G. (1967). "Statistical Methods," 6th ed. Iowa State Univ. Press. Ames.

SNow, M. H. (1977). Myogenic cell formation in regenerating rat skeletal muscle injured by mincing. I. A fine structural study. Anat. Rec. 188, 181-199.

WAYLAND, H., AND Frasher, W. G. (1971). Intravital microscopy on the basis of telescopic principles: Design and application of an intravital microscope for microvascular and neurophysiological studies. Int. Symp. Mod. Technol. Physiol. Sci. (Munich), 125-145.

Weiss, S. W., and Faulkner, J. A. (1983). Revascularization of skeletal muscle transplanted into the Hamster cheek pouch: Electron microscopy. Microvasc. Res. 26, 65-73.

White, T. P., Maxwell, L. C., Sosin, D. M., and Faulkner, J. A. (1981). Capillarity and blood flow of transplanted skeletal muscles of cats. Amer. J. Physiol. 241 (Heart Circ. Physiol. 10), H630-H636. 